\title{
Einstellung eines palatinal verlagerten Eckzahns mittels Alignertherapie - ein Fallbericht
}

\section{Insertion of a Palatally Displaced Canine Using Aligner Therapy - A Case Report}

\author{
Autoren \\ Julia Funke1, Werner Schupp², Julia Haubrich², Britta A. Jung ${ }^{1}$
}

Institute

1 Klinik für Kieferorthopädie, Universität Freiburg

2 Praxis für Kieferorthopädie, Dres. Schupp/ Haubrich, Köln

Schlüsselwörter

Eckzahnretention, Alignertherapie, Auxiliaries

Key Words

Impacted canines, Aligner therapy, auxiliaries

\author{
Bibliografie \\ Inf Orthod Kieferorthop 2020; 52: 259-265 \\ DOI 10.1055/a-1242-0044 \\ ISSN 0020-0336 \\ (C) 2020. Thieme. All rights reserved. \\ Georg Thieme Verlag KG, Rüdigerstraße 14, \\ 70469 Stuttgart, Germany
}

Korrespondenzadresse

Dr. Julia Funke

Klinik für Kieferorthopädie

Hugstetter Straße 55

79106 Freiburg

Deutschland

julia.funke@uniklinik-freiburg.de

\section{ZUSAMMENFASSUNG}

Ein günstiger Zeitpunkt für die Einstellung eines potentiell verlagerten Eckzahnes liegt vor dem eigentlichen Durchbruchszeitpunkt, in dem bspw. mit einer Milchzahnextraktion bzw.
Lückenöffnung für den bleibenden Zahn ein Pfad zur passiven Eruption geschaffen werden kann. Geschieht dies nicht oder bleibt der Zahn verlagert, so entsteht eine komplexe Aufgabe, die dem kieferorthopädischen Behandler eine genaue Planung abverlangt und in interdisziplinärer Zusammenarbeit mit einem oralchirurgisch tätigen Zahnarzt, Oral- oder Mund-KieferGesichtschirurgen erfolgen muss.

Das moderne kieferorthopädische Behandlungsspektrum bietet vielerlei Möglichkeiten für die Einstellung verlagerter Zähne. Für den folgenden Patientenfall zeigen wir eine Möglichkeit zur Einstellung eines verlagerten Eckzahns mittels Aligner-Therapie in Kombination mit zusätzlichen adäquaten Hilfsmitteln (Auxiliaries).

\section{ABSTRACT}

An effective date to treat a potentially impacted canine is usually before the actual time of eruption, by extracting the deciduous tooth or opening the gap for the permanent tooth and herewith creating a path for passive eruption.

If this does not happen or if the tooth remains impacted, a complex orthodontic task arises that requires precise planning from the orthodontic practitioner and must be carried out in interdisciplinary collaboration with an oral or maxillofacial surgeon.

The modern orthodontic treatment spectrum offers many possibilities for the regulation of displaced teeth. In the following patient case, the therapeutic approach to an impacted canine using aligner therapy in combination with additional adequate auxiliaries is shown.

\section{Einleitung}

Verlagerte Eckzähne stellen für den Kieferorthopäden immer wieder eine Herausforderung dar. Generell liegt die Prävalenz dieser Problematik zwischen 1 und $3 \%$ in der westlichen Population, wobei Mädchen doppelt so häufig betroffen sind wie Jungen und der obere Eckzahn im Verhältnis zum unteren 10-mal häufiger verlagert ist $[1,2]$. Am häufigsten sind obere Eckzähne palatinal verlagert (50\%), gefolgt von der bukkalen (30\%) und der im Alverolarkamm (20\%) liegenden Verlagerung [3]. Unter Ausgrenzung der dritten Molaren macht der Oberkiefer-Eckzahn $60 \%$ aller verlagerten Zähne aus, davon treten $8 \%$ der Verlagerungen beiderseitig auf [1].

Der Durchbruchspfad des oberen Eckzahns ist sowohl im zeitlichen als auch im räumlichen Sinn der längste [4]. Seitlich der Apertura piriformis in der Fossa canina angelegt, nimmt er einen kurvenförmigen Verlauf vom basalen Kieferabschnitt bis zum Zahnbogen. Zunächst schräg liegend, erfolgt schließlich eine senkrechte 
Einreihung in den Zahnbogen [5]. In der Regel sollte der Durchbruch im Alter von 11-12 Jahren erfolgen.

Ätiologisch ist eine palatinale Eckzahnverlagerung eher durch endogene Faktoren begünstigt, wohingegen die Bukkalverlagerung häufig als Folge eines primären oder sekundären Engstands auftritt. Weitere Faktoren, die mit einer Eckzahnverlagerung assoziiert sind, sind Syndrome, Durchbruchshindernisse (Zysten, überzählige Zähne), Milchzahnretentionen sowie verfrühter Verlust eines Milchzahns, Lippen-Kiefer-Gaumenspalten, Ankylosen oder Dilazerationen [6].

Diagnostisch sollten beim Verdacht auf eine Eckzahnverlagerung folgende Hinweise beachtet werden:

- Platzmangel, Mittellinienabweichung, Distalkippung des oberen seitlichen Schneidezahns (besonders bei einseitiger Eckzahnverlagerung, nicht zu verwechseln mit physiologi-
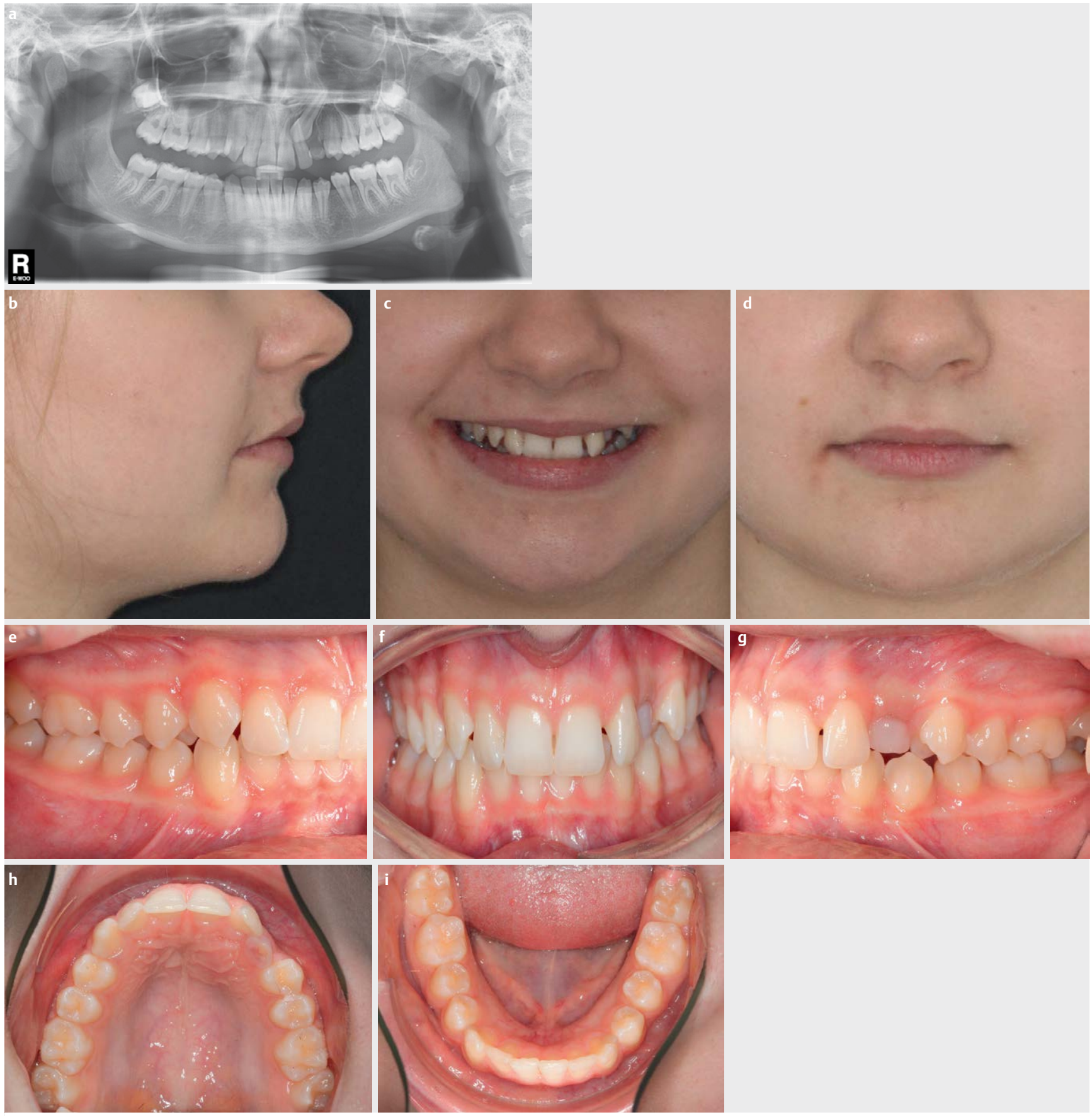

- Abb. 1 Radiologischer a und klinischer Ausgangsbefund b einer 13 Jahre alten Patientin: Es zeigten sich zahlreiche persistierende Milchzähne. Das Orthopantomogramm zeigt die Anlagen der Weisheitszähne 18, 28 und 38. Zahn 48 war zu diesem Zeitpunkt noch nicht röntgenologisch nachweisbar. Kiefergelenke, Kieferhöhlen und weitere knöcherne Strukturen zeigten sich radiologisch ohne pathologischen Befund. Der Zahn 23 war retiniert und verlagert. Zahn 63 war klinisch noch in situ. 
schem „ugly duckling“), Nichtanlage und Zahnanomalien des OK-2ers [7], Milcheckzahnpersistenz ohne Lockerung

- Im OPG spricht ein vergrößerter Winkel zwischen Eckzahnachse und Spina nasalis anterior $\left(\geq 28,4^{\circ}\right)$ ab dem Alter von 9 Jahren für eine Verlagerung [8].

- Palpierbarkeit ca. ab dem 10. Lebensjahr [9].

Zur genauen Lagebestimmung ist in der Regel eine zweite Ebene bzw. eine 3-dimensionale Aufnahme notwendig [10]. Die Risiken bei Nichtbehandlung einer Eckzahnverlagerung sind ein ektopischer Durchbruch oder das Verbleiben im Kiefer sowie pathologische Wurzelresorptionen an den Nachbarzähnen. Hier sind am häufigsten die lateralen Inzisiven betroffen (80\%). Die Resorptionen im Zusammenhang mit verlagerten Eckzähnen sind progressiv und können nur durch Umlenken des Eckzahnes aufgehalten werden [6].

Therapeutisch kann die Extraktion des Milcheckzahnes vorgenommen werden, sobald die Wurzel des bleibenden Zahnes zur Hälfte bis 2/3 entwickelt ist. Voraussetzung ist, dass normale Platzverhältnisse vorliegen und keine Resorptionen an der Schneidezahnwurzel vorgefunden werden. 78\% der verlagerten Eckzähne brechen nach früher Extraktion der Milcheckzähne normal durch [8].

Bleibt der Spontandurchbruch aus, besteht mit einer breiten Palette von Behandlungsalternativen in der Kieferorthopädie die Mög- lichkeit, die verlagerten bleibenden Zähne später einzustellen, sofern diese nicht ankylosiert sind [6].

Mit der folgenden Patientenvorstellung soll dargestellt werden, wie eine solch komplexe Behandlungsaufgabe mittels Alignertherapie gelöst werden kann.

\section{Fallbericht}

Die Patientin ( $\triangleright$ Abb. 1) stellte sich nach Überweisung durch den Hauszahnarzt im Alter von 13 Jahren erstmalig in der kieferorthopädischen Fachpraxis Dres. Schupp/ Haubrich in Köln zum Beratungsgespräch vor. Hauptanliegen der Patientin war der nach distal gekippte Zahn 22 ( $\mathbf{A b b}$. 1f). Allgemeinmedizinisch lagen keine pathologischen Befunde vor, das CMD Short-Screening [11] erwies sich als negativ ( $<2$ positive Befunde).

Nach der Erstellung diagnostischer Unterlagen ließ sich folgende Diagnose zusammenfassen:

Permanente Dentition im Ober- und Unterkiefer mit Ausnahme des Zahnes 23, welcher sich in der Panoramaschichtaufnahme (PSA) als verlagert darstellte ( $\triangleright$ Abb. $\mathbf{1 a}$ ) und an dessen Stelle der Milcheckzahn 63 ( $\triangleright$ Abb. 1g) persistierte. In der Panoramaschichtaufnahme (PSA) stellten sich zudem die Anlagen der Weisheitszähne 18, 28 und 38 dar. Zahn 48 war zu diesem Zeitpunkt noch nicht a
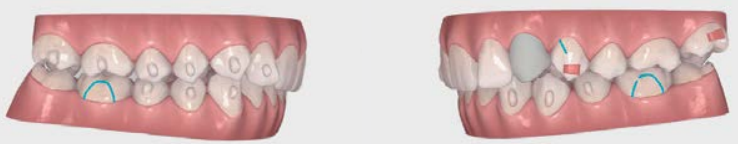

b
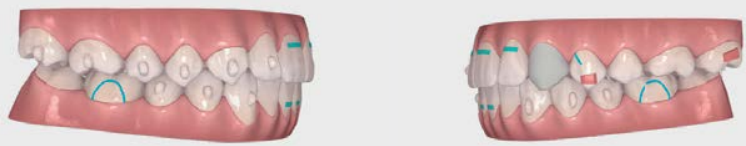
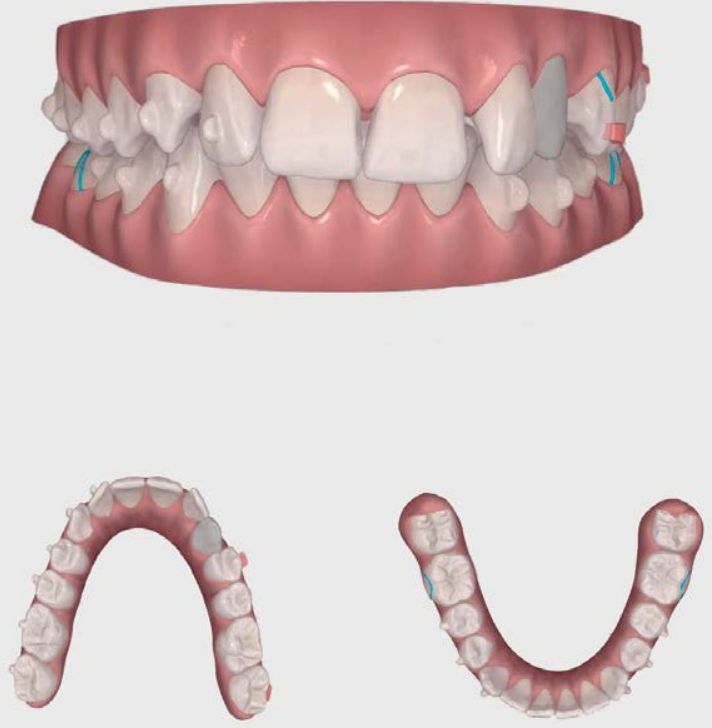
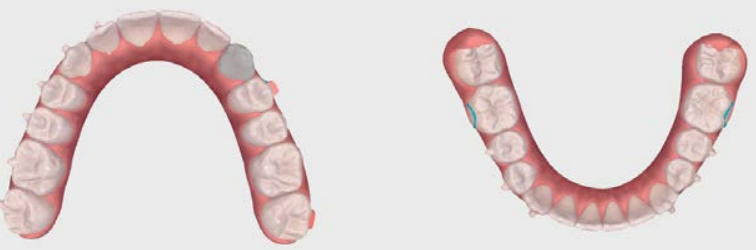

- Abb. 2 Das digitale Malokklusionsmodell in der in der Clincheck-Software a vor der Behandlung und $\mathbf{b}$ nach digitaler Behandlungsplanung. 
röntgenologisch nachweisbar. Kiefergelenke, Kieferhöhlen und weitere knöcherne Strukturen zeigten sich radiologisch ohne pathologischen Befund ( $\triangleright$ Abb. 1a).

Der Oberkiefer imponierte durch eine steil und eng stehende Front sowie Einzelzahnabweichungen (Dreh- und Kippstände). Im Unterkiefer zeigten sich neben diversen Einzelzahnabweichungen (Dreh- und Kippstände) ein frontaler Engstand und Lücken im Seitenzahnbereich, sowie eine ausgeprägte Spee-Kurve ( $\triangleright$ Abb. 1e-i).

Die Bisslagebestimmung ergab eine Distalokklusion(1/2 Pb) auf der rechten Seite sowie eine Neutralokklusion links. Diese Asymmetrie wurde untermauert durch eine gnathische Mittellinienverschiebung um 2,5 mm nach links. Es lag im Sinne einer Klasse-II/2Verzahnung ein tiefer Biss mit dentaler Abstützung vor (Overbite: $4 \mathrm{~mm}$, Overjet: $1 \mathrm{~mm}$ ).

\section{Behandlungssystematik und -therapie}

- Abb. 2 zeigt den Vergleich zwischen Malokklusionsmodell ( $\triangleright$ Abb. 2a) und dem in der Clincheck Software simulierten Be- handlungsende ( $>$ Abb. 2b). Im Rahmen der Therapie wurde zunächst der Zahn 63 als mögliches Durchbruchshindernis für den Zahn 23 entfernt. Es folgte die tranversale und sagittale Ausformung der Zahnbögen im Ober- und Unterkiefer mit dem InvisalignSystem. Hierzu wurden vor dem intraoralen Scan vertikale Attachments im direkten Verfahren auf die Zähne 17-12, 33-35 und 4345, sowie ein Hook auf den Zahn 13 modelliert ( $\triangleright$ Abb. $3 a-e)$.

Beim Einsetzen der ersten Aligner wurde zusätzlich ein Knöpfchen auf Zahn 36 und 46 ergänzt, sowie Verankerungsattachments auf 24 und 27 und ein Precision Cut auf Zahn 24. Die sequentielle, durch intermaxilläre Klasse-II-Gummizüge unterstützte Distalisierung der oberen Seitenzahnreihen diente zum Platzgewinn im Frontzahnbereich und insbesondere zur weiteren Öffnung des Durchbruchspfades des Zahnes 23. Die Aufrichtung der Oberkiefer-Frontzähne wurde durch Power Ridges unterstützt. Im Unterkiefer wurden die Spee-Kurve nivelliert und Einzelzahnabweichungen korrigiert. Insgesamt wurden für die erste Behandlungsphase 64 Aligner geplant, die wöchentlich gewechselt wurden.
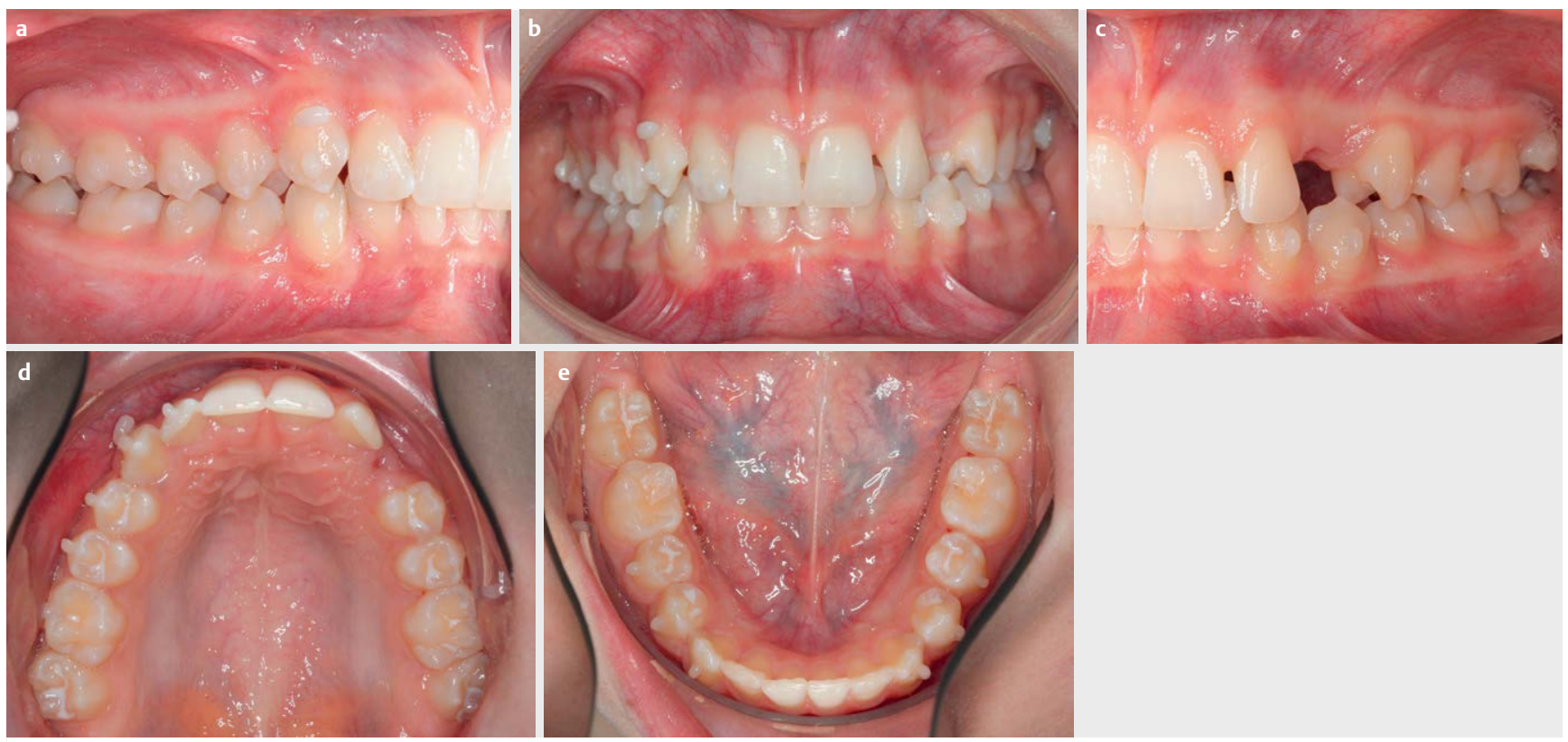

Abb. 3 Klinisch intraoraler Befund nach Extraktion des Zahnes 63 und nach Applizieren der notwendigen Attachments und Hooks im Rahmen der Aligner-Therapie.
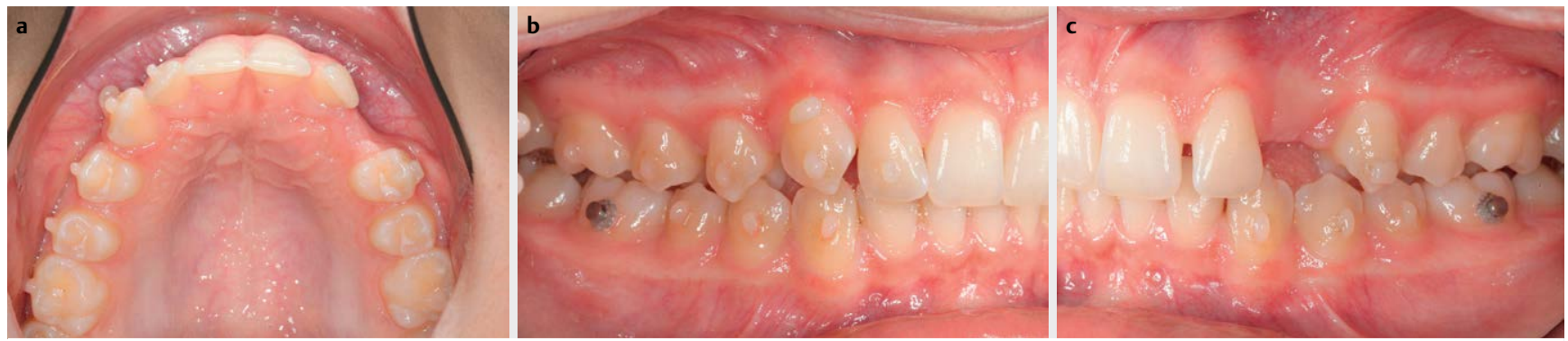

- Abb. 4 Zwischendiagnostik nach der vollständigen Distalisierung von 16 und 15 mit Super-Klasse-I-Molarenverzahnung rechts und links sowie ausreichendem Platzangebot für die Einstellung des Zahnes 23 nach 37 Alignern. 

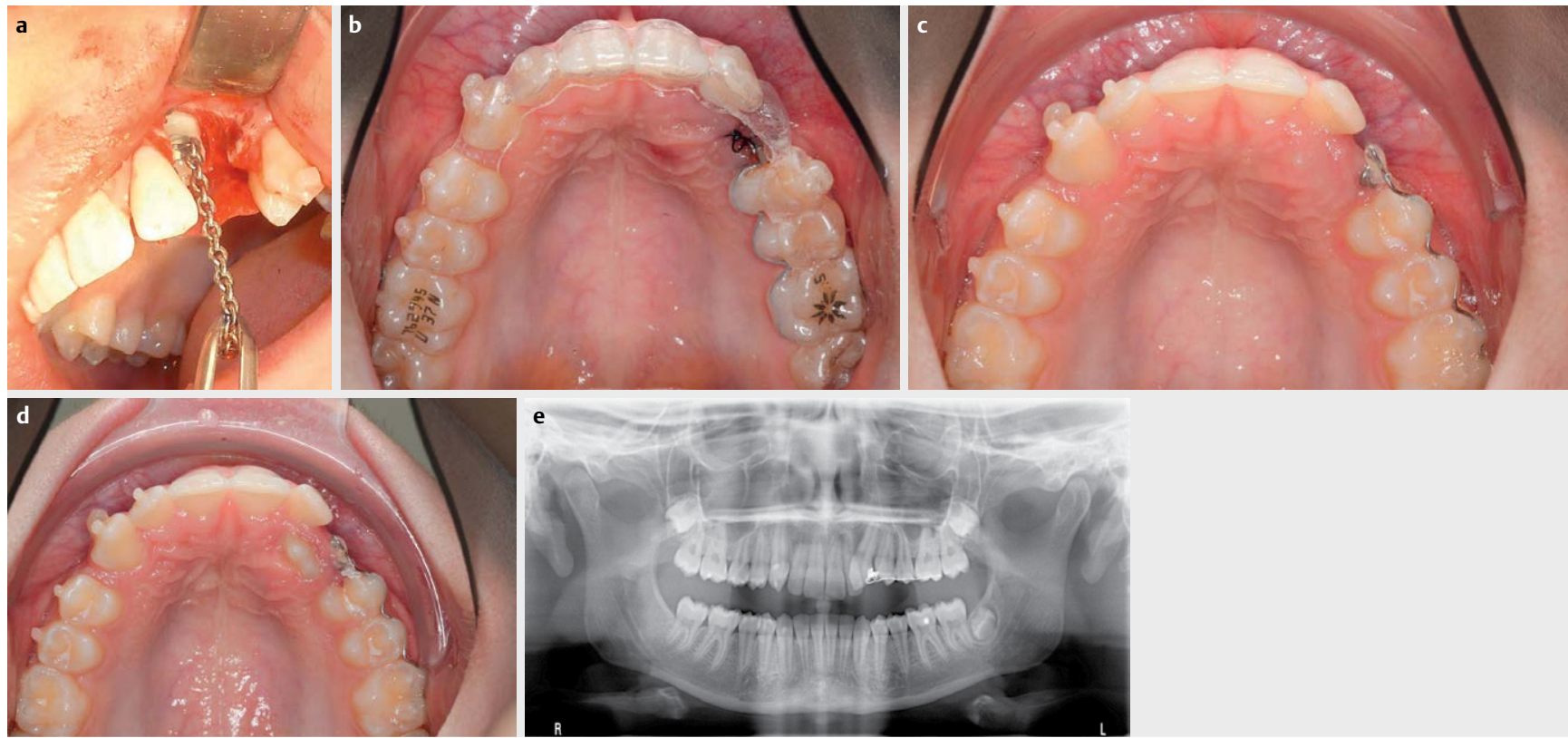

- Abb. 5 a Chirurgische Freilegung und Applikation einer Zugkette, b initiale Extrusion mithilfe eines palatinal befestigten Teilbogens von 24-26 und Aligner 37 in situ; $\mathbf{c}$ und $\mathbf{d}$ Wechsel auf einen bukkal befestigten Teilbogen (regio 24-26) und abschließende Extrusion des Zahnes 23 in die Mundhöhle, e PSA zur Zwischendiagnostik.

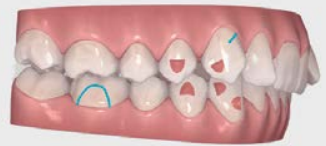

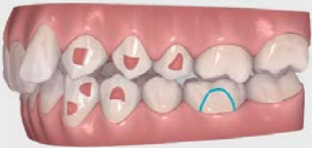
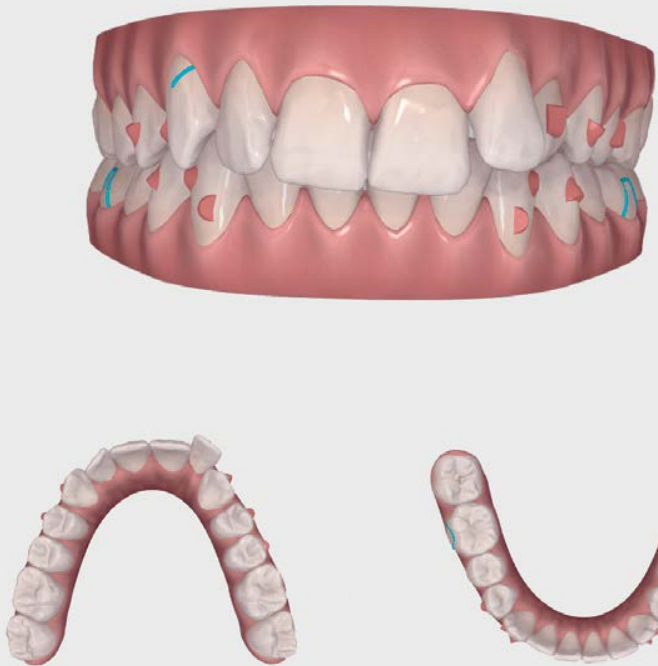

b
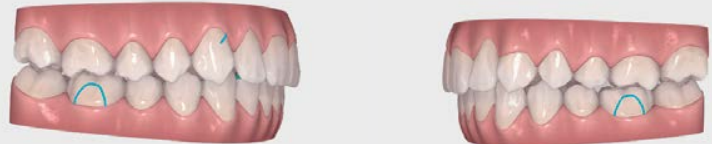

- Abb. 6 Behandlungsplanung im Rahmen eines Case-Refinements in der Clincheck-Software nach Extrusion des Zahnes 23: a digitale Malokklusionsmodelle Ober- und Unterkiefer nach Behandlungspause und Fortsetzung der Behandlung, b nach digitaler Behandlungsplanung. Attachments und Hooks aus a sind in $\mathbf{b}$ zwecks geplanter Retentionsschienen nicht zu sehen. 
Nachdem sich der verlagerte Zahn 23 nach Milchzahnextraktion und weiterer Lückenöffnung durch die sequentielle Distalisierung ( Abb. 4) nach 37 Alignern nicht spontan einstellte, wurde dieser chirurgisch freigelegt ( $\triangleright \mathbf{A b} \mathbf{b}$. $\mathbf{5 a} \mathbf{a}-\mathbf{b}$ ) und mithilfe eines geklebten Teilbogens extrudiert ( $\triangleright \mathbf{A b b} \mathbf{b} \mathbf{5} \mathbf{c} \mathbf{- e}$ ). Die Aligner wurden in dieser Zeit nicht weiter gewechselt und zur Retention des bisher erzielten Behandlungsergebnisses nachts weitergetragen.

Nach Durchbruch in die Mundhöhle wurde eine Behandlungspause eingelegt, da die Patientin für 10 Monate ins Ausland ging. Ihr Wunsch war, in dieser Zeit die Attachments zu entfernen. Nach ihrer Rückkehr wurde ein erneuter Intraoralscan durchgeführt und die Attachments in der Clincheck-Software neu eingeplant ( $\triangleright$ Abb. 6). Auch der Zahn 23 konnte nun mit einem Attachment versehen werden. Es wurden 38 zusätzliche Aligner hergestellt, um die Zahnbögen final auszuformen. Um eine Feineinstellung der Okklusion in Klasse I zu erzielen, wurden erneut Button Cut Outs auf den Zähnen 36 und 46, sowie ein Precision Cut auf 13 und ein Hook auf 23 ergänzt und Klasse-II-Gummizüge getragen. Auf den Zähnen 12-22 wurden palatinale Bite Ramps zur Korrektur des tiefen Bisses virtuell geplant ( $\triangleright$ Abb. 6).
Insgesamt wurde die Patientin mit 75 Alignern, also 75 Wochen aktiv mit Alignern behandelt. Hinzu kommen die Phase der Extrusion des Zahnes 23 sowie die Behandlungspause aufgrund des Auslandsaufenthaltes. Insgesamt betrug so die Zeit von der Erstvorstellung bis zum Behandlungsabschluss 3 Jahre ( $\mathbf{A b b}$. 7-8). Zur Retention trägt die Patientin nachts im Oberkiefer eine herausnehmbare Schiene (Biolon, Fa. Dreve 1,5 mm), im Unterkiefer wurde lingual ein festsitzender Retainer von 34-44 inseriert.

\section{Diskussion}

Die Alignertherapie hat sich innerhalb der vergangenen 2 Jahrzehnte in der Kieferorthopädie etabliert. Während sich das Indikationsspektrum zunächst auf geringe Lücken- und Engstände beschränkte [12], werden heute, 20 Jahre nach Einführung des Invisalign-Systems, zunehmend auch komplexere Behandlungen bei Erwachsenen sowie Kindern und Jugendlichen durchgeführt. Korrekturen in der Transversal-, Sagittal- und auch Vertikalebene sind bei präziser Planung unter Berücksichtigung der biologischen Gegebenheiten des Patienten und der biomechanischen Prinzipien in der kieferorthopädischen Behandlung möglich [13].
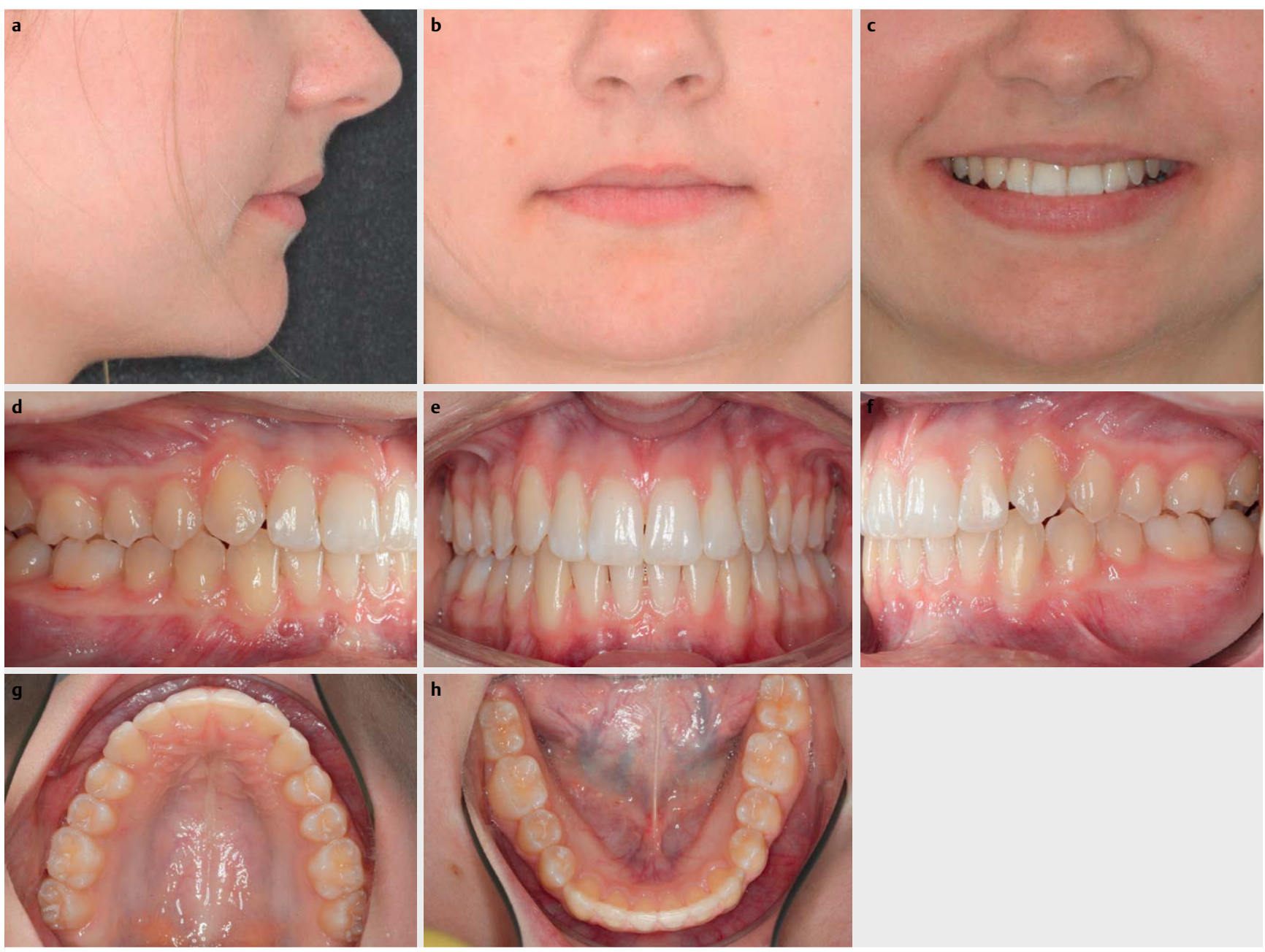

Abb. 7 Klinisches Endergebnis mit ausgeformten Zahnbögen und Klasse-I-Verzahnung mit physiologischem Overjet und Overbite sowie korrigierter Mittellinie. 


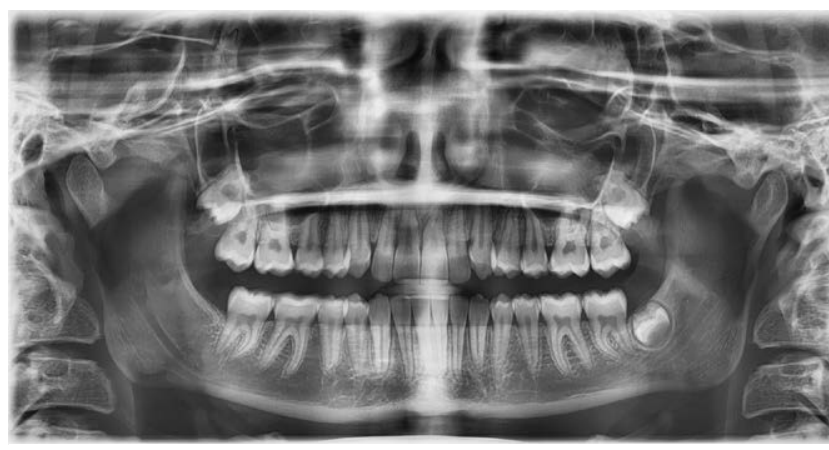

- Abb. 8 Panoramaschichtaufnahme nach Behandlungsabschluss und nach Einstellung des retinierten und verlagerten Zahnes 23. Die Extraktion der Weisheitszähne 18, 28 und 38 wurde anschließend empfohlen.

Die Patienten genießen den Vorteil einer nahezu unsichtbaren Behandlung, die zusätzlich mit einer verbesserten Hygienefähigkeit einhergeht, sind auf der anderen Seite aber auch zu einer hervorragenden Compliance aufgefordert, ohne welche eine Alignerbehandlung nicht durchführbar wäre.

Weder die Einordung eines verlagerten Eckzahnes noch die sequentielle Distalisierung oder Mittellinienkorrektur kann mit einem Aligner allein erfolgen. In Verbindung mit geeigneten Hilfsmitteln (Teilbogen, Klasse-II-Gummizüge), wie im zuvor beschriebenen Patientenfall dargestellt, ist es allerdings möglich die Aligner so zu unterstützen, dass zufriedenstellende Behandlungsergebnisse für Behandler und Patient erzielt werden können [14].

Die Verwendung eines direkt gebondeten Teilbogens ist dabei nur eine von mehreren Möglichkeiten, einen Aligner so zu modifizieren, dass ein verlagerter Zahn in Richtung der Okklusionsebene extrudiert werden kann.

Als weitere Verankerungselemente können in Kombination mit Alignern bspw. skelettal verankerte Mechaniken eingesetzt werden, die den Vorteil einer maximalen Verankerung bieten und die Nebenwirkungen auf die Nachbarzähne auf ein Minimum reduzieren [15].

Allerdings ist gerade bei palatinal verlagerten Eckzähnen sorgfältig abzuwägen, ob und wo die Insertion einer Schraube bzw. eines osseointegrierten Implantats möglich ist, ohne dabei den verlagerten Zahn zu verletzen $[16,17]$.

Bei sehr guter Compliance ist es ebenfalls denkbar, dass der Patient selbstständig Gummizüge vom Kettchen über den Aligner spannt [18] (vergleichbar mit einer Platten-Mobilisierungsapparatur [19]), um eine Extrusion zu erzielen.

[1] Gabka J, Kaspar E. Statistische Übersicht über retinierte Zähne. J Orofac Orthop 1975; 36: 331-339

[2] Watted N, Abu-Hussein M, Awadi O et al. Clinical study of impacted maxillary canine in the Arab population in Israel. Int J Public Health Res 2014; $2:$ 64-70

[3] Diedrich P. Die kieferorthopädische Einordnung retinierter Zähne. [Buchverf.] W Ketterl. Deutscher Zahnärzte Kalender. München: Hanser; 1986

[4] Dewel BF. The upper cuspid. Its development and impaction. Angle Orthod 1949; 19: 79-90

[5] Dausch-Neumann D. Der Durchbruchsweg bleibender Eckzähne. J Orofac Orthop 1970; 31: 9-16

[6] Bishara S. Impacted maxillary canines: A review. Am J Orthod Dentofacial Orthop 1992; 101: 159-171

[7] Trauner R, Byloff-Clar H, Stepantschitz E. Die Vererbung und Entwicklung der Zahn- und Kieferstellungsanomalien. J Orofac Orthop 1961; 22: 1-71

[8] Ericson S, Kurol J. Early treatment of palatally erupting maxillary canines by extraction of the primary canines. Eur J Orthod 1988; 10: 283-295

[9] Patel D. Are patients with impacted canines referred too late? $\mathrm{Br}$ Dent J 2016; 221: 561-564

[10] Watted N, Proff P, Reiser V et al. CBCT; In Clinical Orthodontic Practice. J Dent Med Sci (IOSR) 2015; 14: 102-115

[11] Georg Meyer. Short clinical screening procedure for initial diagnosis of temporomandibular disorders. Journal of Aligner Orthodontics (JAO) 2018; 2: 91-98

[12] Boyd RL, Miller RJ, Vlaskavic V. The Invisalign System in Adult Orthodontics: Mild Crowding and Space Closure Cases. JCO 2000; 34: 203-212

[13] Schupp W, Haubrich J. Aligner Orthodontics. Berlin: Quintessence publishing; 2015: 3

[14] Schupp W, Neumann I, Haubrich J. Class II correction with the Invisalign System. JCO 2010; 44: 28-35

[15] Eliseo A, Fiorillo G. Temporary Anchorage Devices (TADs) and Invisalign: a combination that can be used to meet aesthetic expectations in the orthodontic treatment of adult patients. Journal of Aligner Orthodontics 2019; 3: 15-27

[16] Jung BA, Kunkel M, Schmelzeisen R et al. Insertion von Gaumenimplantaten zur skelettalen Verankerung. Inf Orthod Kieferorthop 2018; 50: 165-167

[17] Jung BA, Kunkel M. Skelettale Verankerungselemente - wissenschaftliches Update. Inf Orthod Kieferorthop 2016; 48: 163-169

[18] David Couchat. Traction of impacted teeth with the Invisalign System. Journal of Aligner Orthodontics 2018; 2: 53-58

[19] Gero Kinzinger. Initiale Mobilisierung verlagerter Oberkiefereckzähne mithilfe modifizierter Zugketten und herausnehmbarer Plattenapparatur. Kieferorthop 2018; 32: 65-73

\section{Interessenkonflikt}

Die Autoren geben an, dass kein Interessenkonflikt besteht. 